\section{Molecular Diagnosis and Prenatal Diagnosis in Abelson's Helper Integration 1 gene (AHI1) in a Family with Joubert Syndrome}

\author{
Chen Biliang*, Li Yu, Xu Ying, Zhang Jianfang, Tang Xing, \\ Song Tingting, Guo Fenfen, Xu Hui, Yan Feng and Wang \\ Detang
}

Department of Obstetrics and Gynecology, Xijing Hospital, Fourth Military Medical University, Xi'an 710032, China

\begin{abstract}
Joubert syndrome is a neurologic disorder with abnormalities of the cerebellum and brainstem, and the Molar Tooth Sign (MTS) is the typical clinical feature. This article was to identify potential mutations in a Chinese patient with Joubert syndrome and related disorders and carry out prenatal diagnostic testing for the family by Sanger sequencing. Two variants were found in AHI1 gene by sequencing of 20 Joubert-related genes for the Chinese proband at a time, and one of the variants (c.2386_c.2380delTTTC) is novel. Sanger validation shows the 2 variants in AHI1 were inherited from each parents, separately. We did prenatal diagnosis for the proband's mother when she was pregnant for 18 weeks, and the data shows the fetus is a carrier. This data shows the importance of the Sanger in clinical diagnosis and the prenatal diagnosis.
\end{abstract}

Keywords: AHI1; Joubert syndrome; Molecular diagnosis; Novel mutation; Prenatal diagnosis

\section{Introduction}

Joubert syndrome (JS, MIM [213300]) was first described in a family in 1968 by Dr. Joubert and his colleagues [1]. The Molar Tooth Sign (MTS) is the key characteristic of JS, it shows unique complex midbrain-hindbrain malformation, appeared in nearly all cases [2]; other diagnostic features include hypotonia in infancy, cerebellar ataxia, intellectual disability and developmental delay, abnormal eye movements and/or breathing pattern $[3,4]$. According to some literature, autistic has been a comparatively common component of JS [5]. Joubert Syndrome and Related Disorders (JSRD) encompass

${ }^{*}$ Corresponding author: Chen Biliang, Department of Obstetrics and Gynecology, Xijing Hospital, Fourth Military Medical University, No. 127, Changle Road (West), Xin Cheng District, Xi'an city, Shaanxi province, China. Tel: 862984771236 ; Fax: 8629 84771236; E-mail: xyfck1010@aliyun.com

Citation: Biliang C, Yu L, Ying X, Jianfang Z, Xing T, et al. (2016) Molecular Diagnosis and Prenatal Diagnosis in Abelson's Helper Integration 1 gene (AHI1) in a Family with Joubert Syndrome. J Reprod Med Gynecol Obstet 1: 003.

Received: July 1, 2016; Accepted: Aug 9, 2016; Published: Aug 23, 2016 classic JS and additional features. JSRD are divided into six types [6]: (1) classic JS; (2) JS with ocular defect (JS-O); (3) JS with renal defect (JS-R); (4) JS with oculorenal defects (JS-OR); (5) JS with hepatic defect (JS-H, or $\mathrm{COACH}$ syndrome); (6) JS with orofaciodigital defects (JS-OFD, or OFDVI syndrome). The prevalence of JS and JSRD has been estimated at approximately 1:100,000 [7].

According to data, JSRD can be inherited in an autosomal recessive manner and an X-linked manner, and some genes associates with JSRD which are common in clinic are listed in the table 1 (Table 1, Gene Reviews, NCBI). Based on these genes, less than half of the mutations with JSRD can be identified. As a result, molecular diagnosis of these patients and their family are very important.

\begin{tabular}{|c|c|c|c|c|}
\hline JBTS & Gene/Protein & Variation ratio & Locus & $\begin{array}{l}\text { Numbers of } \\
\text { exons }\end{array}$ \\
\hline JBTS-1 & INPP5E & unknown & $9 q 34.3$ & 10 \\
\hline JBTS-3 & AHI1 & $7 \%-10 \%$ & $6 q 23.2$ & 29 \\
\hline JBTS-4 & NPHP1 & $1 \%-2 \%$ & $2 q 13$ & 20 \\
\hline JBTS-5 & CEP290 & $10 \%$ & $12 q 21.33$ & 54 \\
\hline JBTS-6 & TMEM67 & $10 \%$ & $8 q 22.1$ & 32 \\
\hline JBTS-7 & RPGRIP1L & $2 \%-4 \%$ & $16 q 12.2$ & 27 \\
\hline JBTS-8 & ARL13B & $<1 \%$ & $3 q 11.1$ & 12 \\
\hline JBTS-9 & CC2D2A & $10 \%$ & $4 q 15.33$ & 38 \\
\hline JBTS-10 & OFD1 & rare & Xp22 & 26 \\
\hline JBTS-2 & TMEM216 & $3 \%$ & $11 q 13.1$ & 6 \\
\hline JBTS-15 & CEP41 & $<1 \%$ & $7 q 32$ & 12 \\
\hline JBTS-14 & TMEM237 & $<1 \%$ & $2 q 33$ & 13 \\
\hline JBTS-17 & C5Orf42 & unknown & $5 \mathrm{p} 13$ & 55 \\
\hline JBTS-12 & KIF7 & unknown & $15 q 26.1$ & 21 \\
\hline JBTS-13 & TCTN1 & unknown & $12 q 24.11$ & 19 \\
\hline Unassigned & TCTN2 & unknown & $12 q 24.31$ & 18 \\
\hline JBTS-18 & TCTN3 & unknown & $10 q 24.1$ & 14 \\
\hline JBTS-16 & TMEM138 & unknown & $11 q 12.2$ & 6 \\
\hline JBTS-20 & TMEM231 & unknown & $16 q 23.1$ & 7 \\
\hline JBTS-11 & TTC21B & unknown & $2 q 24.3$ & 30 \\
\hline
\end{tabular}

In this article, we discussed a Chinese patient of JSRD and the prenatal diagnosis for the proband's family. This proband has been actually diagnosed JSRD. We used Sanger to identify variants in this proband and found compound heterozygosity for one novel mutation in exon 18 of gene AHI1:c.2386_c.2380delTTTC and one known mutation in exon 8 of gene AHI1:c.910_911insA.

\section{Methods}

\section{Case report}

In the department of Obstetrics and Gynecology of the first affiliated hospital of The Fourth Military Medical University, we received a Chinese family with JS proband. The proband's mother was pregnant for 18 weeks (Figure 1a), when she visited our hospital. The proband with clinical manifestations of Joubert syndrome got 
supportive brain imaging in the Department of Radiology, Maternal and Child Health Hospital, in Shanxi province.

The proband, a three months old male Chinese baby, who was born at term naturally without any complications, was the first child of his healthy parents. His mother denied consanguinity with her husband and had no history of teratogen exposure during or immediately preceding pregnancy.

Clinical examination was undertaken when the boy is 3 months old and the boy was died when he is six months old. Hypotonia and abnormal right eye movement is observed. In the mean time Developmental delay was also noticed. Magnetic Resonance Imaging (MRI) of the brain showed Cerebellar Vermis Hypoplasia (CVH), deepened interpeduncular fossa, thick and elongated superior cerebellar peduncles, abnormally shaped fourth ventricle and hypoplastic vermis. (Figure 1b). All the features showed classical MTS, which are the key diagnostics criteria of JSRD clinically.

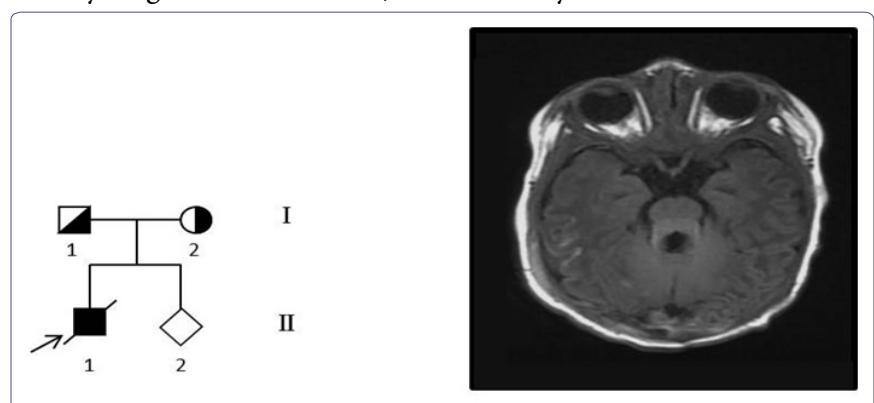

$1 \mathrm{a}$

1b

Figure 1: 1a. Pedigree drawing of the proband's family. 1b. MRI image of the brain of the proband with Joubert syndrome showing a typical Molar Tooth Sign (MTS).

After obtaining written informed consent from this Chinese family, umbilical cord tissue was collected from the boy and $5 \mathrm{~mL}$ of peripheral blood was collected from his parents and $10 \mathrm{~mL}$ amniotic fluid during 18 weeks of pregnancy and 2ML cord blood during 23 weeks of pregnancy of the mother was collected. DNA samples were prepared following the manufacturer's standard procedures (QIAamp DNA Blood MiNi Kit; Qiagen, Hilden, Germany).

\section{Targeted next-generation sequencing and variants verify}

A custom capture array was designed to capture all exons (449), splice sites, and the immediately adjacent introns sequences of the 20 JS and JSRD-related genes. The target exon capture, enrichment, and elution were performed following the protocols with minor modifications (Roche Nimble Gen, Inc.). The successive pooling, hybridization and sequencing processes were performed, followed by previous instructions described in the literature [8]. As a result, 158 $\mathrm{kb}$ of the targeted exon sequences plus the flanking $10 \mathrm{bp}$ into the introns of the targeted genes were specifically captured and enriched. BWA software (Burrows-Wheeler Aligner) was employed to perform the read mapping.

In order to verify the data obtained by targeted next-generation sequencing, primers were designed to amplify the coding exons (429 ) and exon-intron junctions in AHI1 by polymerase chain reaction. The PCR product was sequenced (ABI 3730 DNA sequencer), and the data was analyzed with Sequencing Analysis (Human Gene Mutation Database, HGMD).

\section{Results}

In this study, the exons of 20 genes related to Joubert syndrome and related disorders were recognized by next generation sequencing and detected by DNA sequencing. Maternal effect has been eliminated by Short Tandem Repeat (STR). Approximately $158 \mathrm{~kb}$ of the "target region" for the proband were captured and sequenced. The average coverage is $99.58 \%$ for the family, and the average sequencing depth is 463.25-fold.

Only two potential mutations in AHI1 were detected from the proband: c.910_911insA in the exon 8 and c.2323_2326delGAAA in the exon 18, and both of them are in the coding region. Because no more DNA of the proband can verify the result offered by targeted next-generation sequencing. Sanger sequencing technology was used to analyze the identification of potential mutations in the parents and reveal the inheritance background of JSRD for this family (Figures $2 \mathrm{a} \& \mathrm{~b}$ ). According to the analysis, the truncating mutation (c.910_911insA) was inherited from the proband's mother, which has been reported [9]. The frame shift (c.2323_2326delGAAA) was found both in the proband and his father, which is novel. The result shows that the proband's parents are both carriers.



Figure 2 a: Sanger sequencing verification of the mutations identified by next-targeting sequence.

b: The predicated full-length human Joubert in protein gives a 1196-aa message with WD repeats and $\mathrm{SH} 3$ domain. The arrows indicate the relative position of the mutations detected.

The DNA extracted from amniotic fluid cells were amplified by PCR and detected by DNA sequencing. The data shows all the JS and JSRD-related genes have been amplified and analyzed and the fetus inherits thec.910_911insA in the exon 8 from her mother. DNA extracted from cord blood validates our testing result.

\section{Discussion}

In our study, we identified the genetic base of this Chinese male baby with Joubert syndrome, and found compound heterozygosity for one novel mutation in gene AHI1, and carry out the prenatal diagnosis for the proband's family. The genes caused JS and JSRD uncover two major phenomenon: (1) all the genes coding for proteins of the primary cilium were known as "ciliopathies"; (2) some genes were concerned with the clinical phenotypes. So far, AHI1 is the most commonly mutated gene and has only found in the JSRD [10-13], nearly $10-15 \%$ of all patients have mutations in AHI1, and associated with autism [5]. The most frequently feature in AHI1-realated JSRD 
is retinal dystrophy existing in almost $80 \%$ patients with mutations in AHI1. According to the data, this proband should be JSRD instead of classical JS.

AHI1 mRNA is expressed highly in brain and kidney in human fetal tissue, and in cerebellum and cerebral cortex in human adult, respectively. To the contrary, it is expressed weakly in liver and lung of human fetal tissue and in the putamen of human adult, separately [14]. AHI1, which encodes cytoplasmic adaptor protein, is composed of an N-terminal coiled-coil domain, seven WD40 repeats and one SH3 domain [15].

The truncating mutation, c.910_911insA (p.Thr304AsnfsX6) in exon 8 , which was first described by a Duth research group, causes frame shift and damaging effect on AHI1 protein function. Retinal disease, which also performed in this Chinese boy, showed in nearly 76\% in AHI1-related JSRD patient [9, 11-14]. Because of the deletion identified in exon 18 of the gene AHIl causes frame shift and premature stop codon, it was predicted to be destructive. These four-base deletion (c.2323_2326delGAAA) in the exon 18 truncating the protein at amino acid 802 (p.Glu795LeufsX8) and also eliminating the WD repeats and $\mathrm{SH} 3$ domain.

Because of the fetus is a carrier of JSRD, genetic counseling should be carried out when the baby grow up, get married and be ready to have a baby. As molecular diagnosis is the precise criterion of JS and JSRD, we suggest all JS and JSRD-related genes mutation analysis should be performed in prenatal diagnosis, even if the pathogenic mutation of the proband have been identify. This is an effective mean to avoid the result of false-positive or false-negative.

In this case, the combination of target gene capture, next-generation, polymerase chain reaction and Sanger sequencing were applied in order to verify the gene mutations in the Chinese male baby with Joubert syndrome and conduct the prenatal diagnosis for his family. Our finding gives a description of the variation in gene AHI1, includes one novel mutation, and indicted the importance of molecular diagnosis and prenatal diagnosis for the families with recessively inherited disorders.

\section{Reference}

1. Joubert M, Eisenring JJ, Andermann F (1968) Familial dysgenesis of the vermis: a syndrome of hyperventilation, abnormal eye movements and retardation. Neurology 18: 302-303.
2. Maria BL, Hoang KB, Tusa RJ, Mancuso AA, Hamed LM, et al. (1997) "Joubert syndrome” revisited: key ocular motor signs with magnetic resonance imaging correlation. J Child Neurol 12: 423-430.

3. van Dorp DB, Palan A, Kwee ML, Barth PG, van der Harten JJ (1991) Joubert syndrome: a clinical and pathological description of an affected male and a female fetus from the same sibship Am J Med Genet 40: 100-104.

4. Chance PF, Cavalier L, Satran D, Pellegrino JE, Koenig M, et al. (1999) Clinical nosologic and genetic aspects of Joubert and related syndromes. J Child Neurol 14: 660-672.

5. Alvarez Retuerto AI, Cantor RM, Gleeson JG, Ustaszewska A, Schackwitz WS, et al. (2008) Association of common variants in the Joubert syndrome gene (AHI1) with autism. Hum Mol Genet 17: 3887-3896.

6. Valente EM, Dallapiccola B, Bertini E (2013) Joubert syndrome and related disorders. Hand Book Clin Neurol 113: 1879-1888.

7. Yachnis AT, Rorke LB (1999) Neuropathology of Joubert syndrome. J Child Neurol 14: 655-659.

8. Wei X, Ju X, Yi X, Zhu Q, Qu N, et al. (2011) Identification of sequence variants in genetic disease-causing genes using targeted next-generation sequencing. PLoS One 6: 29500.

9. Kroes HY, van Zon PH, Fransen van de Putte D, Nelen MR, Nievelstein RJ, et al. (2008) DNA analysis of AHI1, NPHP1 and CYCLIN D1 in Joubert syndrome patients from the Netherlands. Eur J Med Genet 51: 24-34.

10. Parisi MA (2009) Clinical and molecular features of Joubert syndrome and related disorders. Am J Med Genet C Semin Med Genet 151: 326-340.

11. Dixon-Salazar T, Silhavy JL, Marsh SE, Louie CM, Scott LC, et al. (2004) Mutations in the AHI1 gene, encoding jouberin, cause Joubert syndrome with cortical polymicrogyria. Am J Hum Genet 75: 979-987.

12. Utsch B, Sayer JA, Attanasio M, Pereira RR, Eccles M, et al. (2006) Identification of the first AHI1 gene mutations in nephronophthisis-associated Joubert syndrome. Pediatr Nephrol 21: 32-35.

13. Valente EM, Brancati F, Silhavy JL, Castori M, Marsh SE, et al. (2006) AHI1 gene mutations cause specific forms of Joubert syndrome-related disorders. Ann Neurol 59: 527-534.

14. Ferland RJ, Eyaid W, Collura RV, Tully LD, Hill RS, et al. (2004) Abnormal cerebellar development and axonal decussation due to mutations in AHI1 in Joubert syndrome. Nat Genet 36: 1008-1013.

15. Jiang X, Hanna Z, Kaouass M, Girard L, Jolicoeur P (2002) Ahi-1, a novel gene encoding a modular protein with WD40-repeat and $\mathrm{SH} 3$ domains, is targeted by the Ahi-1 and Mis-2 provirus integrations. J Virol 76: 9046-9059. 